\title{
Endoscopic extraperitoneal radical prostatectomy after radical resection of pT1-pT2 rectal cancer: a report of thirty cases
}

\author{
Zhuo Liu, Dechuan Li, Yinbo Chen \\ Department of Oncological Surgery, Zhejiang Cancer Hospital, Hangzhou, China
}

Videosurgery Miniinv 2017; 12 (1): 68-74

DOI: https://doi.org/10.5114/wiitm.2017.66475

\begin{abstract}
Introduction: Endoscopic extraperitoneal radical prostatectomy (EERPE) has gained popularity for the treatment of localized prostate cancer. However, prior complex lower abdominal or pelvic surgery can complicate subsequent EERPE. To date, there have been few reports on patients who underwent EERPE after radical resection of PT1-pT2 rectal cancer.

Aim: To present our experience with EERPE in patients after radical resection of pT1-pT2 rectal carcinoma and introduce a simple and effective way to create an extraperitoneal working space.

Material and methods: Thirty patients after radical resection of pT1-pT2 rectal carcinoma were treated with EERPE for biopsy-proven localized prostate cancer. Operation time, estimated blood loss, conversion to open surgery rate, transfusion rate and transurethral catheter time were recorded. Meanwhile, functional outcome (continence and potency) and oncological outcome were reviewed.

Results: The average operative time was $168 \mathrm{~min}$. Mean blood loss was $195 \mathrm{ml}$. There was no need for conversion to open surgery or transfusion. The catheter was removed on postoperative day (POD) 7.8. After a mean follow-up time of 53.1 months, 3 patients had a prostate-specific antigen level relapse over $0.1 \mathrm{ng} / \mathrm{ml}$. At the follow-up time, 26 patients were completely continent, and 4 needed 1-2 pads/day. Of the 6 patients who underwent neurovascular bundle preservation, none have experienced return of erections at the last follow-up time.

Conclusions: Endoscopic extraperitoneal radical prostatectomy after radical resection of rectal carcinoma appears promising, with feasibility in experienced hands. The operative data, postoperative urinary incontinence and oncological outcomes appear encouraging, but the rate of erectile dysfunction seems to be disappointing.
\end{abstract}

Key words: laparoscopy, extraperitoneal, radical prostatectomy, radical rectal resection.

\section{Introduction}

Endoscopic extraperitoneal radical prostatectomy (EERPE) has gained popularity for the treatment of localized prostate cancer [1], since it preserves peritoneal integrity, minimizes the risk of inadvertent adjacent bowel injury [2] and decreases the rate of postoperative trocar point hernia [3].

Previous abdominal surgery promotes adhesion formation and is traditionally regarded as a relative contraindication to laparoscopic radical prostatectomy [4]. Al-Shareef et al. [5] reported 32 cases of extraperitoneal robot-assisted laparoscopic radical prostatectomy (e-RALP) after laparoscopic prosthetic mesh inguinal herniorrhaphy and recommended that e-RALP was a viable surgical alternative for patients in that clinical situation. Stolzenburg et al. [6] summarized their experience of 857 patients with previous abdominal or pelvic surgery. They concluded that EERPE was feasible for those patients. Nevertheless, 
prior complex lower abdominal or pelvic surgery can complicate subsequent EERPE. To date, there have been few reports on patients who underwent EERPE after radical resection of pT1-pT2 rectal cancer.

\section{Aim}

The aim of the study was to present our experience of 30 patients who underwent EERPE after radical resection of $\mathrm{pT} 1$ - $\mathrm{pT} 2$ rectal carcinoma and introduce a simple, safe and effective way to create an extraperitoneal working space in patients with prior pelvic surgery.

\section{Material and methods}

\section{Patients}

From January 2001 to December 2013, thirty patients with previous radical rectal resection underwent EERPE in our medical center. The mean prostate specific antigen (PSA) level was 13.42 (7.44-27.53) $\mathrm{ng} / \mathrm{ml}$. The mean prostate volume was 40.2 (23-72) g. Twenty-four patients had primary low anterior resec- tions (LAR) of RO stage, and the others had abdominoperineal resection (APR) of RO stage. The median interval time from the previous surgery was 6.3 (4.5-15) years. No patients received peroperative radiotherapy. Patients with LAR had transrectal ultrasound guided biopsies and patients with APR had transperineal ultrasound guided biopsies, which showed prostate adenocarcinoma. Pelvic lymph node dissection (PLND) was routinely performed in patients with a serum PSA level of greater than $10 \mathrm{ng} / \mathrm{ml}$ or a preoperative Gleason sum of 7 or greater. All the patients were staged with abdominal and pelvic computed tomography and bone scintigraphy to rule out metastatic disease. The patients were followed up at the $3^{\text {rd }}$ month postoperatively and every 6 months thereafter for 2 years, then in yearly intervals.

\section{Surgical technique}

The laparoscope was placed upon the incision after direct dissection to the posterior sheath of the rectus abdominis (Photo $1 \mathrm{~A}$ ). The sutured peritoneum had severe adhesions to the abdominal wall
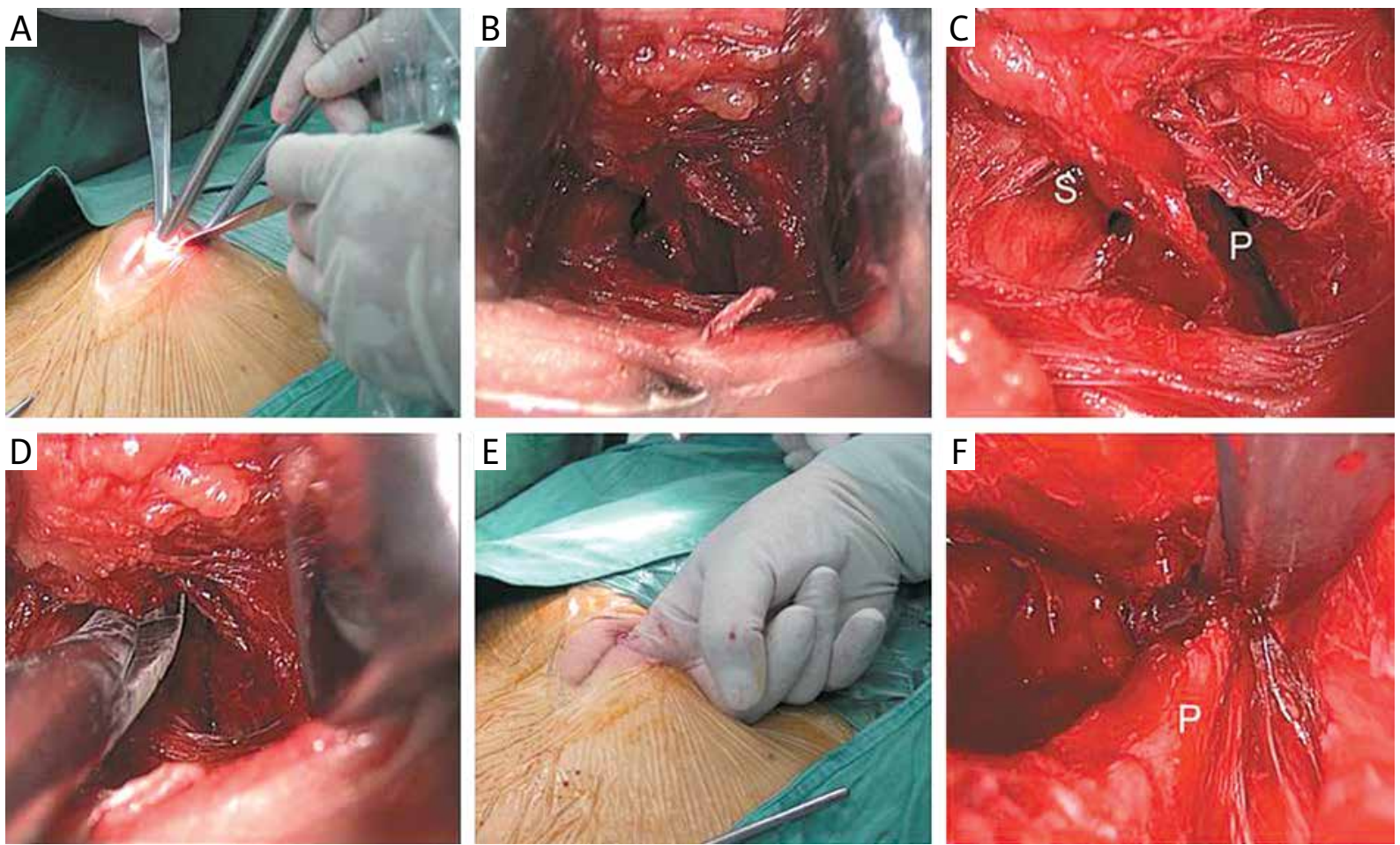

Photo 1. A - External view of a $30^{\circ}$ optical system placed upon the incision. B, C - Endoscopic view of the initial access space set up anterior to the peritoneum (P) beside the prior scar (S). D - Endoscopic view of releasing the adhesions with tissue scissors. $\mathbf{E}$ - Finger dissection to release the loose adhesions and enlarge the approach space. $\mathbf{F}$ - Further sharp dissection after blunt finger dissection to divide the peritoneum (P) from the abdominal wall 
(Photos 1 B, C). Tissue scissors was inserted beside the laparoscope to release the adhesive peritoneum (Photo 1 D). Finger dissection should be carried out at the time to release the loose adhesions and enlarge the approach space (Photo 1 E). Meanwhile, the two skin retractors could be advanced upwards to provide as much space between the peritoneum and abdominal wall as possible. Dissection was performed until the peritoneum was nearly divided from the abdominal wall (Photo $1 \mathrm{~F}$ ). After that, balloon dilation was carried out to develop the space of Retzius. Considering that the dense pelvic adhesions might divide the pelvic region, balloon dilation was performed toward different directions to create enough right/left pelvic space (Figures $1 \mathrm{~A}, \mathrm{~B}$ ). In 1 patient, the right hypogastric region could not be enlarged effectively. Thus, another similar access was set at the lateral side of the scar to enlarge the remaining right hypogastric region (Figures $1 \mathrm{C}, \mathrm{D}$ ).

Since an initial preperitoneal space had been set up, the other trocars could be placed routinely under endoscopic view. Patients with previous APR might present with left port-site adhesions due to the colostomy which should be completely removed with the help of laparoscopic adhesiolysis through the opposite working port. Adhesions were encountered in all procedures independently of the patients' history. The patients with APR did not carry the risks of rectal injury, which could save time of dissection of the prostatic posterior surface. Neurovascular bundle (NVB) preservation was performed in 6 patients.

Patient demographics, preoperative data, pathologic data, and functional outcome (continence and potency) were recorded for each patient. The intraoperative and postoperative complications were classified according to the Clavien classification. Postoperative evaluation of continence and erectile function was performed using the International Continence Society (ICS) questionnaire and the international index of erectile function along with sexual encounter profile (SEP) diaries, respectively. Complete continence was described as the absence of need for pads, total incontinence as the need for more than 2 pads/ day and incontinence as the need for 1-2 pads/day.

\section{Statistical analysis}

The biochemical recurrence-free survival (BCRFS) rate was estimated using the Kaplan-Meier method. The analysis was performed using Statistical Pack- age for the Social Sciences v.13 (SPSS, Chicago, IL, USA). All 30 procedures were performed by two experienced surgeons (each with experience of $>300$ EERPES).

\section{Results}

Demographic and perioperative data are presented in Table I. All of the procedures were completed successfully. There was no conversion to open surgery.

The operative time was $168(95-261) \mathrm{min}$. The total estimated blood loss was 195 (100-420) ml and no need for transfusion was reported. Finally, the pathologic results came as prostate adenocarcinoma with Gleason grade $4+4(n=2)$, Gleason grade $4+3$ $(n=6)$, Gleason grade $3+4(n=14)$, Gleason grade $3+3(n=8)$, and pathologic stage $\mathrm{pT}_{\mathrm{a}} \sim \mathrm{pT} 3_{\mathrm{b}}$. The data are presented in Table II. One patient developed symptomatic lymphocele, Clavien Grade III, which was confirmed by ultrasonography. The patient was managed by percutaneous drainage, and the body temperature returned to the normal level after that. All patients had cystography to exclude the fistula before removal of the catheter. The catheter was removed on postoperative day (POD) 7.8 (5-13). They were discharged from hospital on POD 10.8 (8-17).

Mean follow-up time was 53.1 (1.7-115) months. Six patients with $\mathrm{pT} 2 \mathrm{c} \sim 3 \mathrm{c}$ were found to have biochemical recurrence at the follow-up time of $8,12.3$, 42.5, 56.2, 88.6, and 97.8 months. They were all consequently treated with antiandrogens. The Kaplan-Meier estimate for the 60-month probability of BCRFS was $80.9 \%$ (Figure 2).

At 3 months postoperatively, 10 (33.3\%) patients were continent, 14 (46.7\%) used 1-2 pads/day, and $6(20.0 \%)$ used more than 2 pads/day. At 6 months, $18(60.0 \%)$ of the patients were continent, 8 (26.7\%) used $1-2$ pads/day, and 4 (13.3\%) used more than 2 pads/day. At 12 months, 24 (80.0\%) of the patients were continent, 2 (6.7\%) used 1-2 pads/day, and 4 (13.3\%) used more than 2 pads/day. At the last follow-up time, 26 (86.7\%) patients were continent and only $4(13.3 \%)$ patients used $1-2$ pads/day. No potency was observed postoperatively. The details are presented in Table III.

\section{Disscusion}

Prior abdominal surgery is a predictor of adhesions, and previous autopsy studies revealed that 
A

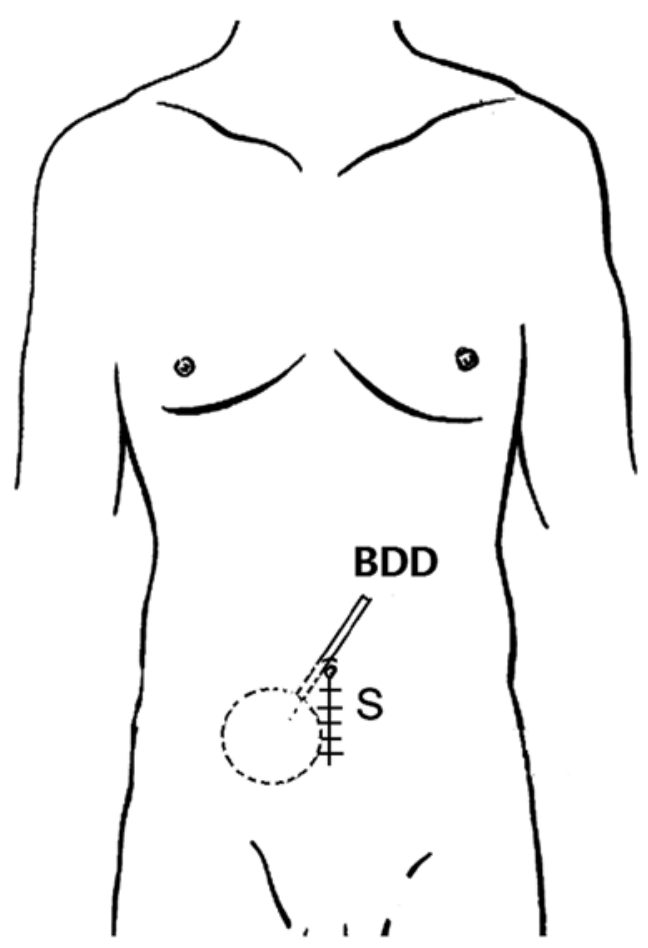

C

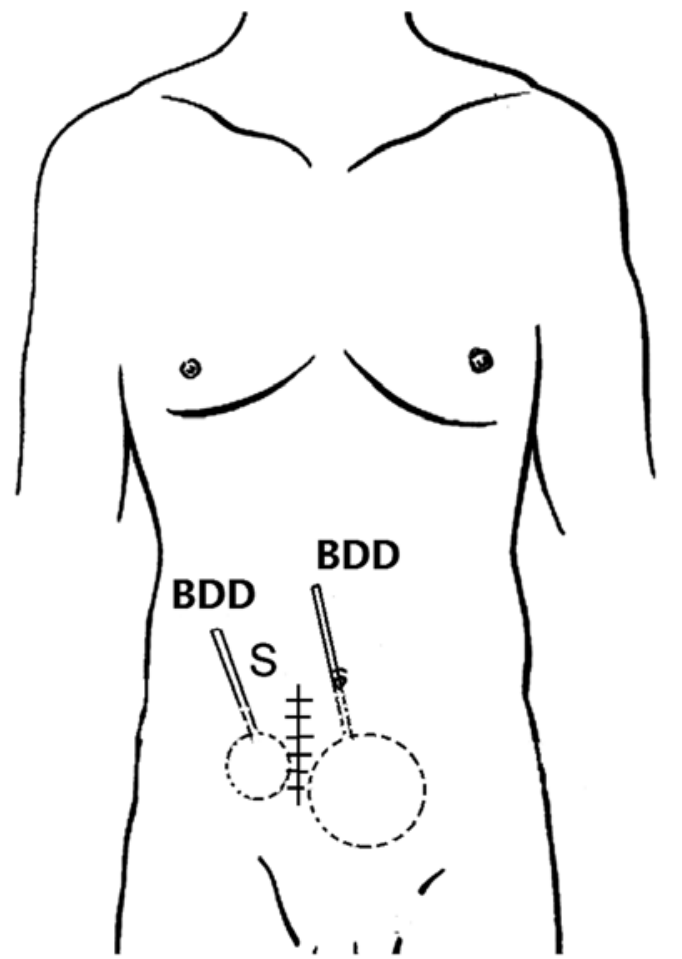

B

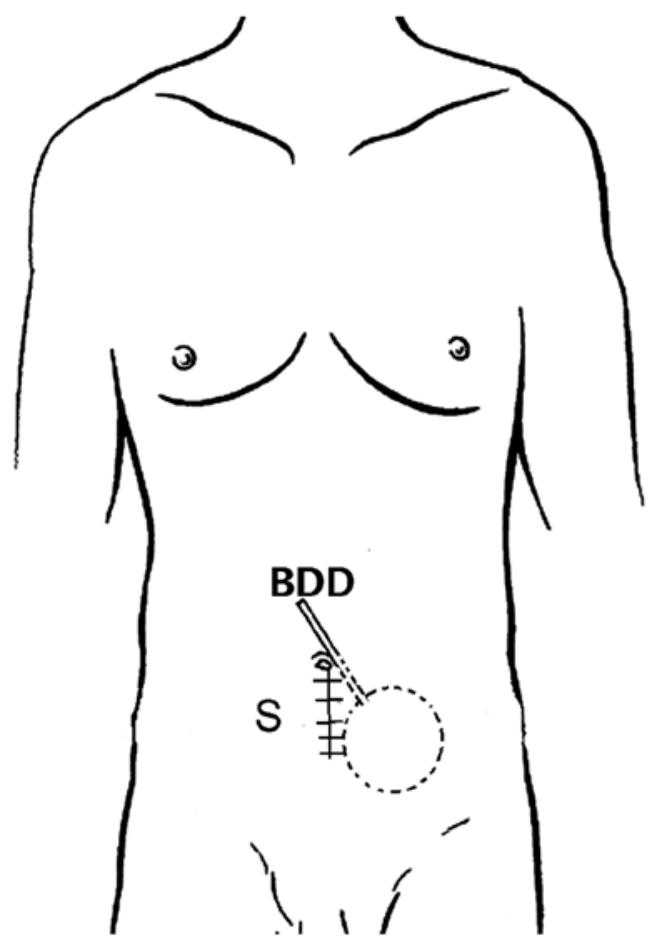

D

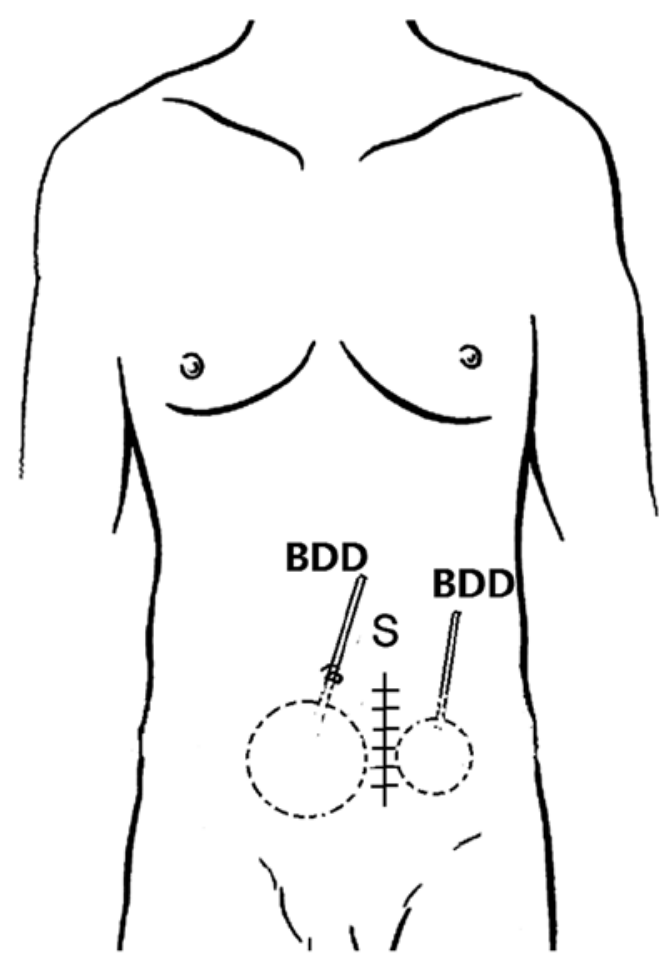

Figure 1. A, B - The balloon dilator device (BDD) is placed at the right/left side of the prior scar (S) to dilate the extraperitoneal space effectively. C, D - If the right/left region cannot be dilated effectively, extra balloon dilation can be performed through another similar access created at the lateral side of the scar 
Table I. Perioperative data $(n=30)$

\begin{tabular}{|c|c|}
\hline Parameter & Result \\
\hline Age [years] & 66.3 (range: 62-74) \\
\hline $\mathrm{BMI}\left[\mathrm{kg} / \mathrm{m}^{2}\right]$ & 24.58 (range: $21.12-32.06)$ \\
\hline \multicolumn{2}{|l|}{ Prior rectal resection: } \\
\hline LAR & $24(80.0 \%)$ \\
\hline APR & $6(20.0 \%)$ \\
\hline \multicolumn{2}{|l|}{ Rectal carcinoma pathologic stage: } \\
\hline $\mathrm{T} 1$ & $4(13.3 \%)$ \\
\hline $\mathrm{T} 2$ & $26(86.7 \%)$ \\
\hline Abdominal scar $[\mathrm{cm}]$ & 11 (range: 8-16) \\
\hline $\begin{array}{l}\text { Time from previous rectal } \\
\text { resection [years] }\end{array}$ & 6.3 (range: 4.5-15) \\
\hline PSA [ng/ml] & 13.42 (range: 7.44-27.53) \\
\hline Prostate weight [g] & 40.2 (range: $23-72$ ) \\
\hline $\begin{array}{l}\text { Preperitoneal space set up time } \\
\text { [min] }\end{array}$ & 14.1 (range: 6.5-23) \\
\hline Operative time [min] & 168 (range: 95-261) \\
\hline Blood loss $[\mathrm{ml}]$ & 195 (range: 100-420) \\
\hline Transfusion rate & $0 \%$ \\
\hline Conversion rate & $0 \%$ \\
\hline Nerve sparing performed & $12(40 \%)$ \\
\hline Lymph node dissection & $24(80 \%)$ \\
\hline Duration of catheterization [days] & 7.8 (range: 5-13) \\
\hline Duration of hospitalization [days] & 10.8 (range: 8-17) \\
\hline
\end{tabular}

intra-abdominal adhesions appeared in approximately $90 \%$ of patients after abdominal surgery [7]. Seifman et al. [8] reported that previous abdominal surgery increased the overall risk of transperitoneal laparoscopy and the location of the scar impacted the access complication rate. Although abdominal surgery was regarded as a contraindication for LRP by some investigators, previous abdominal surgery was not seen as a contraindication by others [9]. Currently, a detail analysis of perioperative complications showed no significant effect of previous abdominal surgery on robot-assisted LRP [10]. To our knowledge, there is no available literature on LRP after radical resection of rectal cancer.
Table II. Pathological characteristics of all patients

\begin{tabular}{|lcl|}
\hline Variable & No. patients (\%) & No. positive margins \\
\hline \begin{tabular}{l} 
Gleason sum: \\
\hline 6
\end{tabular} & $8(26.6)$ & \\
\hline 7 & $20(66.7)$ & \\
\hline $8-10$ & $2(6.7)$ & 0 \\
\hline Pathological stage: & 0 \\
\hline T2a & $8(26.7)$ & 2 \\
\hline T2b & $14(46.6)$ & 0 \\
\hline $\mathrm{T} 2 \mathrm{c}$ & $2(6.7)$ & 1 \\
\hline $\mathrm{T} 3 \mathrm{a}$ & $4(13.3)$ & \\
\hline $\mathrm{T} 3 \mathrm{~b}$ & & 0 \\
\hline
\end{tabular}

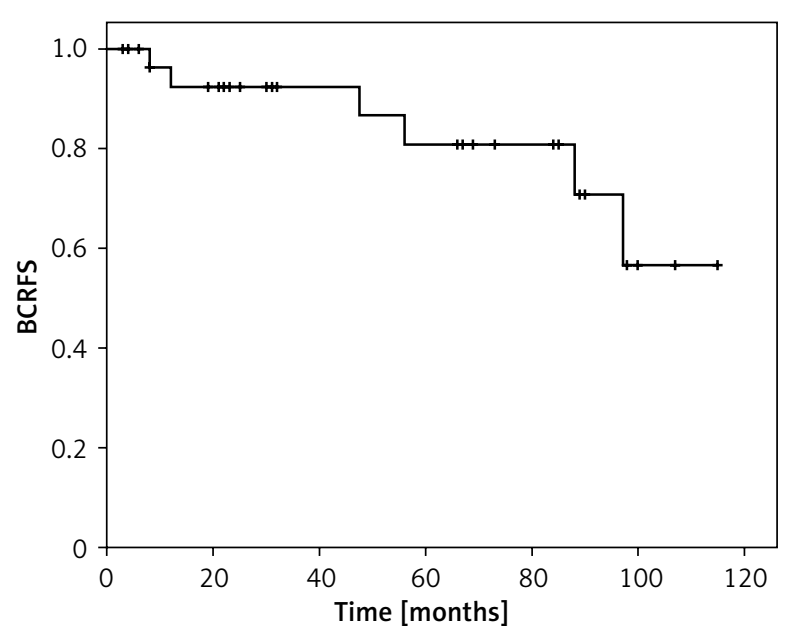

Figure 2. Kaplan-Meier analysis of biochemical recurrence-free survival (BCRFS), with the probability of $80.9 \%$ at 60 months

As for the approach, Boylu et al. [11] summarized their experience on patients with previous abdominal surgery undergoing robotic-assisted laparoscopic radical prostatectomy (RALRP) and noted that the extraperitoneal approach might not be appropriate for patients with previous bowel surgery. Horovitz et al. [12] compared the two approaches for minimally invasive radical prostatectomy and concluded that both methods are safe in patients with prior abdominal surgery. A lower incidence of gastrointestinal complications and shorter length of stay were noted in the extraperitoneal cohort.

Considering the following reasons, we decided to apply an extraperitoneal approach for patients with previous rectal surgery. Firstly, the transperitoneal 
approach on patients with previous rectal surgery may increase the risk of complications because of intra-abdominal adhesions, while the extraperitoneal approach could theoretically decrease those risks. Secondly, EERPE is our routine choice for localized prostate cancer, and we have obtained adequate experience.

We developed a simple method to release the severe adhesions and create an effective extraperitoneal working space. Except for a telescopic camera and tissue scissors, the adhesiolysis did not need any special equipment. So it was convenient and economical. It took a median time of $14.1 \mathrm{~min}$ to set up an effective extraperitoneal working space, which was acceptable. The prostate dissection was similar to the open descending approach: bladder neck dissection, freeing of the seminal vesicles, transectioning of the prostatic pedicles and finally apical dissection. Bladder neck reconstruction was not necessary as it was a bladder sparing technique. The presence of fibrosis made the procedure technically more demanding, and only experienced laparoscopists should perform it.

Stolzenburg et al. [13] described their results of EERPE in 105 patients after open abdominal operation, and the median operative time was $150 \pm 37 \mathrm{~min}$ (range: 90-285 min). However, only three of them had a history of open bowel resection (one underwent hemicolectomy, one underwent abdominoperineal resection, and one underwent sigmoid colectomy). Rodriguez et al. [14] reported their experience of 110 EERPEs after previous pelvic surgery and noted that there was no difference in intraoperative, perioperative and pathological outcomes between patients with or without prior surgery. However, an abundance of preoperative data of EERPE after rectal resection is still rare. In our experience, the mean operative time was $168 \mathrm{~min}$, the total estimated blood loss (EBL) was $195 \mathrm{ml}$, and no cases needed transfusion or conversion to open surgery. Thus, EERPE after radical resection of rectal carcinoma seemed to be associated with acceptable intraoperative morbidity. No thromboembolic events or anastomotic leaks were reported in our small cohort.

Hruza et al. [15] in a study of 500 cases reported that the overall BCRFS rates were $92.9 \%, 78.2 \%$ and $70.6 \%$ at 24, 60 and 120 months after LRP, respectively. Our small cohort indicated a similar BCRFS rate of $80.9 \%$ at 60 months. Stolzenburg et al. [16] previously reported the continence rate of 2000 patients who underwent EERPE, which was 93.2\%. Our
Table III. Postoperative continence results $(n=30)$

\begin{tabular}{|lc|}
\hline Follow-up data & No. patients (\%) \\
\hline 3-mo continence: & $10(33.3)$ \\
\hline 0 pads & $14(46.7)$ \\
\hline $1-2$ pads & $6(20.0)$ \\
\hline >-mo continence: & $18(60.0)$ \\
\hline 0 pads & $8(26.7)$ \\
\hline $1-2$ pads & $4(13.3)$ \\
\hline$>2$ pads & $24(80.0)$ \\
\hline 12-mo continence: & $2(6.7)$ \\
\hline 0 pads & $4(13.3)$ \\
\hline $1-2$ pads & 0 \\
\hline$>2$ pads & $26(86.7)$ \\
\hline At last follow-up continence: & $4(13.3)$ \\
\hline 0 pads & 0 \\
\hline $1-2$ pads & 0 \\
\hline$>2$ pads & 0 \\
\hline 6-mo potency & \\
\hline 12-mo potency & \\
\hline At last follow-up potency & \\
\hline
\end{tabular}

current continence rate was $86.7 \%$ at the last follow-up time. However, the erectile function in our study was disappointing, as none of our patients experienced the return of erections despite NVB preserving procedures being documented in 6 patients.

Several limitations affect the current study, and the results should be carefully interpreted. As previously reported, prior pelvic radiotherapy would increase the difficulty of the procedure of retropubic radical prostatectomy [17] and EERPE needs more technical skills. So we only adopted this minimally invasive procedure in patients with previous rectal cancer staging PT1-pT2, who did not need postoperative radiotherapy. More complicated cases were not involved in our study. Also, the limited number of cases did not allow us to perform reliable statistical analysis to elucidate any statistical significance in terms of preoperative data and functional outcome. More cases and long-term follow-up are required to obtain meaningful results. 


\section{Conclusions}

The EERPE after radical resection of pT1-pT2 rectal carcinoma appeared to be acceptable in intraoperative and perioperative data. The postoperative urinary incontinence and oncological outcomes appear encouraging, but the rate of erectile dysfunction seems disappointing, compared to the patients with standard EERPE.

\section{Conflict of interest}

The authors declare no conflict of interest.

\section{References}

1. Gill IS, Clayman RV, Albala DM, et al. Retroperitoneal and pelvic extraperitoneal laparoscopy: an international perspective. Urology 1998; 52: 566-71.

2. Ploussard G, Xylinas E, Salomon L, et al. Robot-assisted extraperitoneal laparoscopic radical prostatectomy: experience in a high-volume laparoscopy reference centre. BJU Int 2010; 105: 1155-60.

3. Yoshimine S, Miyajima A, Nakagawa K, et al. Extraperitoneal approach induces postoperative inguinal hernia compared with transperitoneal approach after laparoscopic radical prostatectomy. JPN J Clin Oncol 2010; 40: 349-52.

4. Vallancien G, Cathelineau X, Baumert H, et al. Complications of transperitoneal laparoscopic surgery in urology: review of 1,311 procedures at a single center. J Urol 2002; 168: 23-6.

5. Al-Shareef AH, Akin Y, Almouhissen T, et al. Effects of previous hernia repair on extraperitoneal robot-assisted radical prostatectomy: a matched-pair analysis study. J Endourol 2015; 29: 1143-7.

6. Stolzenburg JU, Kallidonis P, Minh D, et al. Endoscopic extraperitoneal radical prostatectomy: evolution of the technique and experience with 2400 cases. J Endourol 2009; 23: 1467-72.

7. Weibel MA, Majno G. Peritoneal adhesions and their relation to abdominal surgery: a postmortem study. Am J Surg 1973; 126: 345-53.

8. Seifman BD, Dunn RL, Wolf JS Jr. Transperitoneal laparoscopy into the previously operated abdomen: effect on operative time, length of stay and complications. J Urol 2003; 169: 36-40.

9. Yumioka T, Iwamoto H, Masago T, et al. Robot-assisted radical prostatectomy in an initial Japanese series: the impact of prior abdominal surgery on surgical outcomes. Int J Urol 2015; 22: 278-82.

10. Tugcu V, Atar A, Sahin S, et al. Robot-assisted radical prostatectomy after previous prostate surgery. JSLS 2015; 19 pii:e2015.00080.

11. Boylu U, Oommen M, Raynor M, et al. Robot-assisted laparoscopic radical prostatectomy in patients with previous abdominal surgery: a novel laparoscopic adhesiolysis technique. J Endourol 2010; 24: 229-32.

12. Horovitz D, Feng C, Messing EM, et al. Extraperitoneal vs. transperitoneal robot-assisted radical prostatectomy in the setting of prior abdominal or pelvic surgery. J Endourol 2017 Jan 10. doi: 10.1089/end.2016.0706.

13. Stolzenburg JU, Ho KM, Do M, et al. Impact of previous surgery on endoscopic extraperitoneal radical prostatectomy. J Urol 2005; 65: 325-31.

14. Rodriguez AR, Kapoor R, Pow-Sang JM. Laparoscopic extraperitoneal radical prostatectomy in complex surgical cases. J Urol 2007; 177: 1765-70.

15. Hruza M, Bermejo JL, Flinspach B, et al. Long-term oncological outcomes after laparoscopic radical prostatectomy. BJU Int 2013; 111: 271-80.

16. Stolzenburg JU, Rabenalt R, Do M, et al. Endoscopic extraperitoneal radical prostatectomy: the University of Leipzig experience of 2000 cases. J Endourol 2008; 22: 2319-25.

17. Masterson TA, Wedmid A, Sandhu JS, et al. Outcomes after radical prostatectomy in men receiving prior pelvic radiation for non-prostate malignancies. BJU Int 2009; 104: 482-5.

Received: 17.10.2016, accepted: 29.01.2017. 